$B-B \cdot T=H_{2}+H_{1} \cdot B$, and $H_{2} \cdot C=0$, contrary to the lemma.

6. Conclusion. In conclusion attention is called to the desirability of clearing up, in the general case, the possibilities for the power of the class of all sets $[H(N)]$ in a compact space for any system $T$, such as has already been done by Mazurkiewicz and Alexandroff (see papers in Fundamenta Mathematicae, vols. 19 and 20) in the special case of the dimensional components. Also a more detailed study of the structure of continua $M$ of varying degrees of connectivity and local connectivity with respect to the sets $H(N)$, in particular in the case* considered in $\$ 5$, would be highly desirable.

The Johns Hopkins University

\title{
INTEGRAL DOMAINS OF RATIONAL GENERALIZED QUATERNION ALGEBRAS $\dagger$
}

\author{
BY A. A. ALBERT
}

1. Introduction. We shall consider generalized quaternion algebras

$$
Q=(1, i, j, i j), \quad j i=-i j, \quad i^{2}=\alpha, \quad j^{2}=\beta,
$$

over the field $R$ of all rational numbers. It is easily shown that, by a trivial transformation on the basis of $Q$, we may take $\alpha$ and $\beta$ to be integers without square factors.

Of great interest in the theory of algebras $Q$ are the integral sets of $Q$. L. E. Dickson $\ddagger$ has called a set $S$ of quantities of $Q$ an integral set if $S$ satisfies the following postulates:

$R$ : The quantities of $S$ have minimum equations with ordinary whole number coefficients and leading coefficient unity.

$C: S$ is closed under addition, subtraction, and multiplication.

$U: S$ contains $1, i, j$.

$M: S$ is maximal.

* A further study of this case is made in the author's paper Cyclicelements of higher order, to appear in the American Journal of Mathematics, vol. 56 (1934).

$\dagger$ Presented to the Society, June 19, 1933.

¥ See Dickson's Algebren und ihre Zahlentheorie, pp. 154-197, for his theory as well as references to the work of Latimer and Darkow. See also Latimer's later paper, Transactions of this Society, vol. 32 (1930), pp. 832-846. 
Using the above definition for $S$, Dickson considered the integral sets for algebras $Q$ with $\alpha=-1$. By a further transformation on the basis of algebras $Q$, Dickson showed, for his case $\alpha=-1$, that $\beta$ could be taken to have no prime factors $p=4 n+1$. He then obtained the sets $S$. Latimer considered algebras $Q$ with $\alpha \equiv \beta \equiv 1(\bmod 2)$, and with $\alpha \equiv 2, \beta \equiv 1(\bmod 8)$ and, after transformations on the basis of $Q$, obtained many sets $S$ for each algebra $Q$. Similar considerations were made by Marguerite Darkow for the case $\alpha \equiv \beta \equiv 2(\bmod 4)$. These latter results of Latimer and Darkow are very complicated and do not complete the problem of finding an integral set for every generalized quaternion algebra.

The above division into special cases is certainly not desirable. Nor is it necessary. For it is obvious that at least an attempt should be made to show that transformations carrying all the cases into a canonical form are possible and it is this canonical form which should be studied.

In the present paper such a canonical form is obtained. It is shown that every rational generalized quaternion algebra is equivalent to an algebra with $\alpha=\tau \equiv 1(\bmod 4)$, where $-\tau$ is a positive prime. The integer $\beta=\sigma$ is also restricted. In particular it is evident that integral sets $S$ should contain the integers of the field $R(i)$ and hence $1, P=(1+i) / 2$, is a basis of such integers. Hence the postulate $U$ is replaced here by

$U^{\prime}: \quad S$ contains $1, P, j$.

But then it is shown that there exist two* $\operatorname{sets} S, \bar{S}$ satisfying $R$, $C, U^{\prime}, M$, and the bases of these sets are explicitly determined.

2. Transformations on Algebras $Q$. Let $R$ be the field of all rational numbers and let $Q$ be any normal simple algebra of degree two over $R$. Then

$$
Q=Q(\alpha, \beta)=(1, i, j, i j), \quad j i=-i j, \quad i^{2}=\alpha, \quad j^{2}=\beta,
$$

where $\alpha \neq 0$ and $\beta \neq 0$ are in $R$. Conversely, every algebra (1) is normal simple.

* Essentially only one set, as we show here that $\sigma=\epsilon \tau+4 \mu^{2}$ and obtain a corresponding set $S$. But then $\sigma=\epsilon \tau+4(-\mu)^{2}$ and $\bar{S}$ is obtained from $S$ by replacing $\mu$ by $-\mu$. 
If we have $i_{0}=\lambda i, j_{0}=(\lambda+\nu i) j$, then $j_{0} i_{0}=-i_{0} j_{0}, i_{0}{ }^{2}=\lambda^{2} \alpha$, $j_{0}^{2}=\left(\mu^{2}-a \nu^{2}\right) \beta$, and $Q=\left(1, i_{0}, j_{0}, i_{0} j_{0}\right)$. Hence we have the following lemma.

LEMma 1. If $\lambda, \mu, \nu$ are rational numbers, then $Q(\alpha, \beta)=Q(\gamma, \delta)$, where $\gamma=\lambda^{2} \alpha, \delta=\left(\mu^{2}-\alpha \nu^{2}\right) \beta$.

Next let $i_{0}=x_{1} i+x_{2} j+x_{3} i j$, so that

$$
i_{0}^{2}=\gamma=f\left(x_{1}, x_{2}, x_{3}\right)=x_{1}^{2} \alpha+x_{2}^{2} \beta-x_{3}^{2} \alpha \beta .
$$

Then I have proved* the following fact.

LEMma 2. If $\gamma=f\left(x_{1}, x_{2}, x_{3}\right) \not \equiv x_{1}^{2} \alpha$ of (2) for rational $x_{1}, x_{2}, x_{3}$, then $Q(\alpha, \beta)=Q(\gamma, \delta)$, where in fact $\delta=-\alpha \beta\left(x_{2}^{2}-x_{3}^{2} \alpha\right)$.

If $\alpha$ is rational, then $\alpha=\rho \epsilon^{-1}$, where $\rho$ and $\epsilon$ are relatively prime integers. Then $\rho \epsilon=\xi^{2} \alpha_{0}$, where $\xi$ and $\alpha_{0}$ are integers and $\alpha_{0}$ has no square factors. If $\alpha_{1}=\xi \epsilon^{-1}$, then $\alpha=\alpha_{1}^{2} \alpha_{0}$ and we call $\alpha_{0}$ the kernel of $\alpha$.

By Lemma 2 any integer having the form $x_{1}^{2} \alpha+x_{2}^{2} \beta-x_{3}^{2} \alpha \beta$ for rational $x_{1}, x_{2}, x_{3}$ may be chosen as a new $\alpha$. Let $\alpha_{0}, \beta_{0}, \gamma_{0}$ be the kernels of $\alpha, \beta,-\alpha \beta$, respectively, so that every value of the form $F(x, y, z)=\alpha_{0} x^{2}+\beta_{0} y^{2}+\gamma_{0} z^{2}$ for integral $x, y, z$ is represented rationally by (2) and may be taken to replace $\alpha$.

Evidently $\gamma_{0}$ is the kernel of $-\alpha_{0} \beta_{0}$, so that not all three of $\alpha_{0}, \beta_{0}, \gamma_{0}$ are even. Moreover, one of $\alpha_{0}, \beta_{0}, \gamma_{0}$ is negative. Either $\alpha_{0} \equiv \beta_{0} \equiv \gamma_{0} \equiv 3(\bmod 4)$, so that $F(1,1,1) \equiv 9 \equiv 1(\bmod 4)$, or one letter, say $a$, is even, and one, say $b$, satisfies the congruence $b \equiv 3(\bmod 4)$, whence $a \cdot 1^{2}+b \cdot 1^{2} \equiv 2+3 \equiv 1(\bmod 4)$.

If $\alpha>0$, then one of $\beta_{0}$ and $\gamma_{0}$, say $b$, is negative, so that $\rho=\alpha+4 b \alpha^{2}=\alpha(1+4 \alpha \beta)<0, \rho \equiv 1(\bmod 4)$, and is a value of $f\left(x_{1}, x_{2}, x_{3}\right)$. Hence we may take $\alpha<0, \alpha \equiv 1(\bmod 4)$.

Let us now apply Lemma 1 to replace $\alpha=\alpha_{1}^{2} \alpha_{0}, \beta=\beta_{1}^{2} \beta_{0}$ by their kernels $\alpha_{0}, \beta_{0}$. This is evidently accomplished when we have $\lambda=\alpha_{1}^{-1}, \mu=\beta_{1}^{-1}, \nu=0$. Hence take $\alpha_{0} \equiv 1(\bmod 4), \alpha_{0}<0$.

Let $\pi$ be the greatest common divisor of $\alpha_{0}, \beta_{0}$, so that $\alpha_{0}=\alpha_{2} \pi, \beta_{0}=\beta_{2} \pi$. Then $\alpha_{2}, \beta_{2}, \pi$ are relatively prime in pairs since $\alpha_{0}$ and $\beta_{0}$ have no square factors. It follows that $\alpha_{0}$ is prime to $\beta_{2}\left(\pi-\alpha_{2}\right)$. But if $\lambda=1, \mu=1, \nu=\pi^{-1}$ in Lemma 1 , then

* See my paper in this Bulletin, vol. 37 (1933), pp. 257-258, for the results implying Lemmas 1 and 2. 
$\gamma=\alpha_{0}, \delta=\left(1-\pi^{-2} \alpha_{2} \pi\right) \pi \beta_{2}=\beta_{2} /\left(\pi-\alpha_{2}\right)$. We use Lemma 1 to replace $\beta_{2}\left(\pi-\alpha_{2}\right)$ by its kernel and thus prove the following theorem.

TheOREM 1. Every normal simple algebra of degree two over $R$ is equivalent to an algebra $Q(\alpha, \beta)$, where $\alpha$ and $\beta$ are relatively prime integers without square factors, $\alpha<0, \alpha \equiv 1(\bmod 4)$.

The quadratic form $\phi=-\alpha x^{2}-4 \beta y^{2}$ has relatively prime coefficients, positive leading coefficient, and discriminant $\Delta=-16 \alpha \beta$. Evidently $\Delta$ is an integral square if and only if $-\alpha \beta=1,-\alpha=\beta=1$, which is impossible since $-\alpha \equiv 3(\bmod 4)$. By the Dirichlet Theorem,${ }^{*} \phi$ represents infinitely many positive primes $p$. Since $-\alpha \beta$ has only a finite number of prime divisors, infinitely many positive primes $p$ represented by $\phi$ are prime to $-\alpha \beta$. If $\tau=-p$, then

$$
\tau=\alpha x^{2}+4 \beta y^{2}, \quad-\alpha \beta x^{2} \equiv(2 \beta y)^{2}(\bmod \tau) .
$$

If $x \equiv 0(\bmod \tau)$, then necessarily $y \equiv 0(\bmod \tau)$, so that $\alpha x^{2}+4 \beta y^{2}$ $=\tau \equiv 0\left(\bmod \tau^{2}\right)$, which is impossible. Hence $x$ is prime to $\tau$, $t x \equiv 1(\bmod \tau)$ for integral $t,-\alpha \beta \equiv(2 \beta y t)^{2}(\bmod \tau)$, and $-\alpha \beta=4 \mu^{2}+\epsilon \tau$ for integral $\mu, \epsilon$. By Lemma 2, with $x_{1}=x$, $x_{2}=y, x_{3}=0$, we have $\delta=-4 \alpha \beta x_{2}^{2}$ whose kernel is $-\alpha \beta$. Hence the following theorem is established.

Theorem 2. Every normal simple algebra of degree 2 over $R$ is equivalent to an algebra $Q(\tau, \sigma)$, where $\sigma_{-}$has no square factors and is prime to $\tau,-\tau$ is a prime, and $\sigma=4 \mu^{2}+\epsilon \tau$ when $\tau<0$ and $\tau \equiv 1(\bmod 4)$, for integral $\mu, \epsilon$.

3. The Removal of Certain Factors of $\sigma$. It is well known that the quadratic form

$$
x^{2}-\tau y^{2}-\rho z^{2}, \quad(\tau, \rho)=1,
$$

is a zero form, for $\tau$ prime to $\rho$, if and only if $\tau$ is a quadratic residue of $\rho$ and $\rho$ is a quadratic residue of $\tau$.

Evidently $\tau$ is a quadratic residue of 2 . But 2 is a quadratic residue of $\tau$ if and only if $\tau \equiv 1(\bmod 8)$. For if $\tau=-t, t$ a prime, then

* See A. Meyer, Journal für Mathematik, vol. 103 (1888), pp. 98-116. 


$$
\begin{array}{r}
(2 \mid \tau)=(2 \mid t)=(-1)^{\left(t^{2}-1\right) / 8}=1, \quad t^{2} \equiv r^{2} \equiv 1(\bmod 16) \\
(4 n+1)^{2} \equiv 8 n+1 \equiv 1(\bmod 16), n \equiv 0(\bmod 2) \\
\tau=8 n_{1}+1
\end{array}
$$

Let $\rho$ be the product of all the odd prime factors $q$ of $\sigma$ such that $\tau$ is a quadratic residue of each such $q$. Let $\rho_{0}=2 \rho$ if $\tau \equiv 1$ $(\bmod 8)$ and $\sigma$ is even, while $\rho_{0}=\rho$ if either $\sigma$ is odd or $\tau \neq \equiv 1$ $(\bmod 8)$. Then $x^{2}-\tau y^{2}-\rho_{0} z^{2}=0$ for integral $x, y$, $z$ not all zero. For $\tau$ is a quadratic residue of $\rho_{0}$, and $\rho_{0}$ is a quadratic residue of $\tau$ if and only if $(\rho \mid \tau)=1$, since $-\tau=t$ is a prime. ${ }^{*}$ But

$$
(\rho \mid \tau)=(\rho \mid t)=(-1)^{e}(t \mid \rho)=(-1)^{f}(\tau \mid \rho)=(-1)^{f}=1,
$$

$$
e=\frac{\rho-1}{2} \cdot \frac{t-1}{2}, \quad f=e+\frac{\rho-1}{2}=\frac{\rho-1}{2} \cdot \frac{t+1}{2},
$$

since $(\tau \mid \rho)=1, t+1=-\tau+1 \equiv-1+1 \equiv 0(\bmod 4)$.

The integer $z \neq 0$, since $x^{2}-y^{2} \tau$ is not a zero form when $-\tau>1$, is a prime. Hence $\rho_{0}=\lambda^{2}-\nu^{2} \tau$ for rational $\lambda, \nu$. Moreover $\rho_{0}=\eta^{2}(\bmod \tau)$, where $\eta$ is prime to $\tau$. Hence $\eta \zeta \equiv 1(\bmod \tau)$ for integral $\zeta$, so that, if $\sigma=\sigma_{0} \rho_{0}$, then $\rho_{0} \sigma_{0} \equiv 4 \mu^{2}(\bmod \tau), \sigma_{0} \equiv 4(\mu \zeta)^{2}$ $(\bmod \tau)$. But $Q(\tau, \sigma)=Q\left(\tau, \sigma_{0}\right)$ by Lemma 1 and the following fact is proved.

THEOREM 3. In Theorem 2 we may take $\tau$ a quadratic nonresidue of every odd prime factor of $\sigma$ and $\sigma$ odd if $\tau \equiv 1(\bmod 8)$.

It is well known that $Q(\tau, \sigma)$ is a division algebra if and only if the form

$$
x_{1}^{2}-\tau x_{2}^{2}-\sigma x_{3}^{2}
$$

is not a zero form. Evidently if (6) is a zero form, then $\sigma>0$. But necessarily if (6) is a zero form, then $\tau$ is a quadratic residue of $\sigma$. Since $\tau$ is a quadratic non-residue of every odd prime factor of $\sigma$, then $\sigma=1,2$. If $\sigma=2$, then (6) implies that 2 is a quadratic residue of $\tau$, so that $\tau \equiv 1(\bmod 8)$, a contradiction of Theorem 3 . Hence $\sigma=1$. Conversely, if $\sigma=1$, then $(j-1)(j+1)=0$ and $Q$ is not a division algebra.

THEOREM 4. The algebras of Theorem 3 are all division algebras except for $\sigma=1$.

${ }^{*}$ For of course $\tau$ is already a quadratic residue of $\rho_{0}$ and $(2 \mid \tau)=1$ in the case where $\rho_{0}$ is even; whence $\tau \equiv 1(\bmod 8)$. 
Consider now the congruence $x^{2} \sigma+y^{2} \tau-z^{2} \equiv 0(\bmod 4 \sigma)$. Then $y^{2} \tau-z^{2} \equiv 0(\bmod \sigma)$. If $q$ is any odd prime factor of $\sigma$ not dividing $y$, then $\tau$ is a quadratic residue of $q$, a contradiction. But $\sigma$ has no square factors so that $\sigma=\sigma_{0}$ or $2 \sigma_{0}$, where $\sigma_{0}$ is odd, $y \equiv 0\left(\bmod \sigma_{0}\right)$ so that $z \equiv 0\left(\bmod \sigma_{0}\right)$. If $\sigma_{1}$ is even, then $\tau \neq \equiv 1$ $(\bmod 8), x^{2} \sigma+y^{2} \tau-z^{2} \equiv 0(\bmod 8)$. But $y^{2} \tau-z^{2} \equiv 0(\bmod 2)$, since $\sigma$ is even. Hence $y \equiv z(\bmod 2)$ so that, since $\tau \equiv 1(\bmod 4)$, we shall have $y^{2} \tau-z^{2} \equiv 0(\bmod 4), x^{2} \sigma \equiv 0(\bmod 4), x \equiv 0(\bmod 2)$, $x^{2} \sigma \equiv 0(\bmod 8)$, and $y^{2} \tau \equiv z^{2}(\bmod 8)$. If $y$ is odd, then $z$ is odd and $y^{2} \tau-z^{2} \equiv \tau-1 \equiv 0(\bmod 8)$, which is a contradiction. Hence $y$ is even, $y \equiv z \equiv 0(\bmod 2)$, and we have proved the following result.

TheOREM 5. The integral congruence $x^{2} \sigma+y^{2} \tau-z^{2} \equiv 0(\bmod 4 \sigma)$ implies that $y \equiv z \equiv 0(\bmod \sigma)$.

4. On Domains of Integrity. Let $D$ be a rational semi-simple algebra of order $n$. A set $S$ of quantities of $D$ is called a domain of integrity if it is closed under addition, subtraction, multiplication. Assume also that the quantities of $S$ satisfy integral equations and that $S$ contains the modulus 1 of $D$. Then if $S$ has order $n$, it is well known* that $S$ has a basis

$$
\omega_{10}, \omega_{20}, \cdots, \omega_{n 0}=1 .
$$

We may evidently write

$$
\omega_{i 0}=\sum_{j=1}^{n} \alpha_{i j} u_{j 0}, \quad(i=1, \cdots, n),
$$

where the $u_{j 0}$ are a basis of $D$, the $\alpha_{i j}$ are rational, and

$$
u_{n 0}=1 \text {. }
$$

Then the matrix $A=\left\|\alpha_{i j}\right\|$ is non-singular and

$$
A=\alpha^{-1}\left\|\beta_{i j}\right\|=\alpha^{-1} B
$$

where $B$ is an integer matrix, $\alpha$ is an integer. If $C=\left\|c_{i j}\right\|$ is an integer matrix of determinant unity, the transformation

$$
\omega_{i 0}^{\prime}=\sum c_{i j} \omega_{i 0}
$$

evidently replaces the $\omega_{j 0}$ by a new basis of $S$. But it is well

\footnotetext{
* See Dickson, Algebren und ihre Zahlentheorie, p. 212.
} 
known $\dagger$ that there exists such a matrix $C$ such that

$$
C B=B_{0}=\left\|\gamma_{i j}\right\|
$$

has elements below the diagonal all zero. Then

$$
\omega_{i}^{\prime}=\alpha^{-1} \sum \gamma_{i j} u_{j 0}=\sum \delta_{i j} u_{j 0} .
$$

But now re-arrange the $u_{i 0}$ so that $u_{i}=u_{n-i, 0}$ and similarly $\omega_{i}=\omega_{n-i}{ }^{\prime}$. Then

$$
\omega_{i}=\sum_{j=1}^{i} a_{i j} u_{j},
$$

with rational $a_{i j}$. In particular

$$
\omega_{1}=a_{11} \cdot 1, \quad\left(a_{11} \text { in } R\right),
$$

satisfies an integral equation so that $a_{11}$ is integral. But unity is in $S$ so that, since each $\omega_{i}$ contains one more basal unit than $\omega_{i-1}$, we evidently must have unity an integral multiple of $\omega_{1}$. Hence $a_{11}=1$.

TheOREM 6. If $D$ is a rational semi-simple algebra of order $n$ and $S$ is a domain of integrity of the nth order in $D$ such that the quantities of $S$ satisfy integral equations and the modulus $u_{1}=1$ of $A$ is in $S$, then $S$ has a basis

$$
\omega_{i}=\sum_{j=1}^{i} a_{i j} u_{j}, \quad\left(\omega_{1}=1, a_{i j} \text { in } R\right),
$$

for $i=1, \cdots, n$.

In particular let $S$ contain the basal quantities of $D$. Then

$$
u_{i}=\sum_{j=1}^{n} \eta_{i j} \omega_{j}, \quad(i=1, \cdots, n),
$$

with integer $\eta_{i j}$. Since the $u_{i}$ are linearly independent in $R$, and $\omega_{n}$ contains $u_{n}$, but no other $\omega_{i},(i<n)$, contains $u_{n}$, we evidently have $\eta_{i n}=0,(i<n)$. Similarly $\eta_{i j}=0,(j=i+1, \cdots, n)$, so that

$$
u_{i}=\sum_{j=1}^{i-1} \eta_{i j} \omega_{j}+\eta_{i i} \omega_{i}
$$

† Ibid., p. 221. 
Again applying the linear independence of the $u_{i}$ to coefficients of the $u_{i}$, we have

$$
1=a_{i i} \eta_{i i}
$$

so that we have proved the following theorem.

THEOREM 7. If $S$ contains the basal quantities of $A$ and if (15) holds we have

$$
a_{i i}=\frac{1}{\alpha_{i i}},
$$

where the $\alpha_{i i}$ are integral.*

Let us consider now a generalized quaternion algebra

$$
Q=(1, i, j, i j), \quad j i=-i j, \quad i^{2}=\tau, \quad j^{2}=\sigma,
$$

with integer $\tau$ and $\sigma$. Then if $S$ is a domain of integrity of $Q$ which contains the quantities $1, i, j$, and hence $i j$, and which is such that the quantities of $S$ satisfy integral equations, then $S$ has a basis

$$
\begin{array}{ll}
\omega_{1}=1, & \omega_{3}=\beta_{1}+\beta_{2} i+\beta_{3} j, \\
\omega_{2}=\alpha_{1}+\alpha_{2} i, & \omega_{4}=\gamma_{1}+\gamma_{2} i+\gamma_{3} j+\gamma_{4} i j,
\end{array}
$$

with rational $\alpha_{i}, \beta_{i}, \gamma_{i}$. Every quantity

$$
x=\delta_{1}+\delta_{2} i+\delta_{3} j+\delta_{4} i j
$$

satisfies

$$
\omega^{2}-2 \delta_{1} \omega+N(x)=0,
$$

where $x$ satisfies an integral equation only for integer

$$
2 \delta_{1}, \quad N(x)=\delta_{1}^{2}-\delta_{2}^{2} \tau-\delta_{3}^{2} \sigma+\delta_{4}{ }^{2} \tau \sigma .
$$

Hence $2 \alpha_{1}, 2 \beta_{1}, 2 \gamma_{1}$, are rational integers. Also $i \omega_{2}=\alpha_{2} \tau+\alpha_{1} i$

* Notice that in fact

$$
\omega_{i}=\alpha_{i i}{ }^{-1}\left(u_{i}-\sum_{j=1}^{i-1} \alpha_{j} \omega_{j}\right)
$$

which may simplify the determination of $\omega_{i}$ after the $\omega_{j}$ are determined. This fact is not used here. 
satisfies an integral equation (22), so that $2 \alpha_{2} \tau$ is integral. Similarly $2 \beta_{2} \tau, 2 \beta_{3} \sigma, 2 \gamma_{2} \tau, 2 \gamma_{3} \sigma, 2 \gamma_{4} \sigma \tau$ are integers. But $\alpha_{2}, \beta_{3}, \gamma_{4}$ are the reciprocals of integers by Theorem 6 . Hence we have proved the following theorem.

THEOREM 8. Let $Q$ be a generalized quaternion algebra (19) over $R$ and let $S$ be a domain of integrity of $Q$ containing $1, i, j, i j$, and with all its quantities satisfying integral equations. Then $S$ has a basis

$$
\left\{\begin{array}{c}
\omega_{1}=1, \quad \omega_{2}=\frac{\xi_{1}}{2}+\frac{1}{\xi_{2}} i, \quad \omega_{3}=\frac{\eta_{1}}{2}+\frac{\eta_{2}}{2 \tau} i+\frac{1}{\eta_{3}} j, \\
\omega_{4}=\frac{\zeta_{1}}{2}+\frac{\zeta_{2}}{2 \tau} i+\frac{\zeta_{3}}{2 \sigma} j+\frac{1}{\zeta_{4}} i j,
\end{array}\right.
$$

where the $\xi_{i}, \eta_{i}, \zeta_{i}$ are integers, and

$$
2 \tau \equiv 0\left(\bmod \xi_{2}\right), \quad 2 \sigma \equiv 0\left(\bmod \eta_{3}\right), \quad 2 \sigma \tau \equiv 0\left(\bmod \zeta_{4}\right) .
$$

5. Two Integral Sets of $Q$. We shall consider generalized quaternion division algebras $Q$ over $R$. Our algebras are therefore algebras of Theorem 4 with $\sigma \neq 1$. Consider the quantities

$$
v_{1}=1, \quad v_{2}=\frac{1+i}{2}, \quad v_{3}=j, \quad v_{4}=\frac{i(2 \mu+j)+\tau j}{2 \tau}
$$

Since $\tau=4 n+1, \sigma=4 \mu^{2}+\epsilon \tau$, we have

$$
v_{3}^{2}=\sigma, \quad v_{2}^{2}=v_{2}+n, \quad v_{4}^{2}=\epsilon n+\mu^{2},
$$

so that the quantities (26) satisfy integral equations.

We study the set $S$ of linear combinations

$$
\sum_{i=1}^{n} \lambda_{i} v_{i}
$$

with integer $\lambda_{i}$. Evidently $S$ satisfies $U^{\prime}$, since $S$ contains 1 , $P=v_{2}, j$. Also $S$ is closed under addition and subtraction. It is evident that, since the basal quantities (26) of $S$ satisfy integral equations, $S$ will satisfy postulates $R$ and $C$ if and only if $S$ is closed under multiplication. In order to prove this closure we need only prove that $S$ contains 

$v_{2} v_{3}, \quad v_{3} v_{2}, \quad v_{2} v_{4}, \quad v_{4} v_{2}, \quad v_{3} v_{4}, \quad v_{4} v_{3}$.

In particular $S$ contains

$$
j=v_{3}, \quad i=2 v_{2}-1, \quad i j=2 \tau v_{4}-2 \mu i-\tau j .
$$

We compute

(31) $\quad v_{3} v_{2}=\frac{j(i+1)}{2}=\frac{(1-i) j}{2}=\left(1-v_{2}\right) v_{3}=v_{3}-v_{2} v_{3}$,

which is in $S$ if and only if $v_{2} v_{3}$ is in $S$. But

$$
v_{2} v_{3}=\frac{(1+i)}{2} j=\tau v_{4}-\mu i-2 \eta j
$$

is in $S$. Also

$$
\left\{\begin{aligned}
v_{4} v_{3} & =\frac{i(2 \mu j+\sigma)+\sigma \tau}{2 \tau}=2 \mu v_{4}+\frac{\sigma-4 \mu^{2}}{2 \tau} i-\mu j+\frac{\sigma}{2} \\
& =2 \mu v_{4}-\mu v_{3}+\frac{\lambda i+\sigma}{2} \\
& =2 \mu v_{4}-\mu v_{3}+2 n \epsilon+\epsilon\left(\frac{1+i}{2}\right) \\
& =2\left(\mu^{2}+n \epsilon\right)+\epsilon v_{2}-\mu v_{3}+2 \mu v_{4}
\end{aligned}\right.
$$

is in $S$, while

$$
v_{3} v_{4}+v_{4} v_{3}=\sigma,
$$

so that $v_{3} v_{4}$ is in $S$.

It remains to prove $v_{2} v_{4}$ and $v_{4} v_{2}$ in $S$. Now

(35) $v_{2} v_{4}=\frac{1+i}{2} \cdot \frac{2 \mu i+i j+\tau j}{2 \tau}=\frac{2 \mu \tau+2 \mu i+2 \tau j+(\tau+1) i j}{4 \tau}$.

Since

$$
\frac{i j}{2 \tau}=v_{4}-\frac{2 \mu i+\tau j}{2 \tau},
$$

we have 


$$
\left\{\begin{aligned}
v_{2} v_{4} & =\frac{\tau+1}{2} v_{4}+\frac{2 \tau j+2 \mu i+2 \mu \tau-(\tau+1)(2 \mu i+\tau j)}{4 \tau} \\
& =(2 n+1) v_{4}+\frac{\tau(1-\tau) j+2 \mu \tau(1-i)}{4 \tau} \\
& =(2 n+1) v_{4}-n v_{3}+\mu v_{2}-\mu
\end{aligned}\right.
$$

in $S$. Finally

$$
\left\{\begin{aligned}
v_{2} v_{4}+v_{4} v_{2} & =v_{2} v_{4}+\frac{2 \mu \tau+2 \mu i+(1-\tau) i j}{4 \tau} \\
& =\mu+\frac{4 \mu i+2 \tau j+2 i j}{4 \tau}=\mu+v_{4}
\end{aligned}\right.
$$

is in $S$, so that $v_{4} v_{2}$ is in $S$. We have therefore proved that $S$ satisfies properties $R, C, U^{\prime}$.

Since $\sigma=4 \mu^{2}+\epsilon \tau$, we have also $\sigma=4(-\mu)^{2}+\epsilon \tau$, so that our above argument by which we proved that $S$ has properties $R$, $C, U^{\prime}$ also proves that the set $\bar{S}$ with basis

$$
\text { (39) } y_{i}=v_{i}, \quad(i=1,2,3), \quad y_{4}=\frac{i(-2 \mu+j)+j \tau}{2 \tau},
$$

also has properties $R, C, U^{\prime}$. Moreover $S$ and $\bar{S}$ are actually distinct. For otherwise $y_{4}$ is in $S, v_{4}+y_{4}-j=\tau^{-1} i j$ is in $S$, whence $-\tau^{-2} \sigma \tau=-\sigma \tau^{-1}$ is an integer, contrary to our hypothesis that $\sigma \not \equiv 0(\bmod \tau)$.

We shall now prove that every set $T$ which satisfies $R, C, U^{\prime}$ is either in $S$ or in $\bar{S}$ and hence each of $S$ and $\bar{S}$ is maximal. We will then have proved $S$ and $\bar{S}$ the only sets satisfying $R, C$, $U^{\prime}, M$.

Let then $T$ satisfy $R, C, U^{\prime}$. Since $T$ is a domain of integrity, it has a basis (24). Hence $\omega_{1}=1$ is in $S, \bar{S}$. Also $\omega_{2}$ is an integer of the algebraic field $R(i)$ and hence, as is well known,* is an integral linear combination of $1, v_{2}$. Hence $\omega_{2}$ is in $S, \bar{S}$.

We next write

$$
\omega_{3}=\frac{\eta_{1}}{2}+\frac{\eta_{2} i}{2} \tau+\frac{1}{\eta_{3}} j, \quad 2 \sigma \equiv 0\left(\bmod \eta_{3}\right) .
$$

* Dickson, loc. cit., p. 149. 
But then

$$
\omega_{3}^{\prime}=\omega_{3}-\eta_{1} v_{2}=\frac{\eta_{2}-\eta_{1} \tau}{2} i+\frac{1}{\eta_{3}} j
$$

is in $T$, since $T$ has property $C$. Hence without loss of generality we may take $\eta_{1}=0$ in (40).

The quantity $\omega_{3}$ must have an integral minimum equation. This equation evidently is

so that

$$
\omega_{3}^{2}=\frac{\eta_{2}^{2} \eta_{3}^{2}+4 \sigma \tau}{4 \tau \eta_{3}^{2}}
$$

$$
\eta_{2}^{2} \eta_{3}^{2}+4 \sigma \tau \equiv 0\left(\bmod 4 \tau \eta_{3}^{2}\right) .
$$

Then $4 \sigma \tau \equiv 0\left(\bmod \eta_{3}{ }^{2}\right)$. Since $\sigma \tau$ has no square factor, we have $4 \equiv 0\left(\bmod \eta_{3}^{2}\right), \eta_{3}= \pm 1, \pm 2$. But then $\eta_{2}^{2} \equiv 0(\bmod \tau)$, so that

Compute

$$
\eta_{2}=\eta \tau, \quad \omega_{3}=\frac{\eta i}{2}+\frac{1}{\eta_{3}} j .
$$

$$
v_{2} \omega_{3}=\left(\frac{1+i}{2}\right)\left(\frac{\eta i}{2}+\frac{1}{\eta_{3}} j\right)=\frac{\eta \tau}{4}+\frac{\eta i}{4}+\frac{j}{2 \eta_{3}}+\frac{i j}{2 \eta_{3}},
$$

which must have integral minimum equation (22). Hence $\eta \tau / 2$ is an integer, so that $\eta=2 \eta_{0}$ is even, $\omega_{3}-\eta_{0} i=\eta_{3}{ }^{-1} j$ is in $T$ and has integral minimum equation $\omega^{2}=\sigma \eta_{3}{ }^{-2}$. Since $\sigma$ has no square factor, we have $\eta_{3}= \pm 1$, and $\omega_{3}=\eta_{0} i \pm j$ is in $S, \bar{S}$ as desired.

We finally consider the quantity $\omega_{4}$. By the above transformation on $\omega_{3}$ we may take $\zeta_{1}=0$ in (21) and write

$$
\omega_{4}=\frac{\zeta_{2} i}{2 \tau}+\frac{\zeta_{3} j}{2 \sigma}+\frac{i j}{\zeta_{4}},
$$

where $2 \sigma \tau \equiv 0\left(\bmod \zeta_{4}\right)$. Then $2 \sigma \tau=\zeta_{4} \zeta_{5}$ and

$$
\omega_{4}=\frac{\zeta_{2} i}{2 \tau}+\frac{\zeta_{3} j}{2 \sigma}+\frac{\zeta_{5} i j}{2 \sigma \tau},
$$

so that

$$
\omega_{4}^{2}=\frac{\zeta_{2}^{2} \tau}{4 \tau^{2}}+\frac{\zeta_{3}^{2} \sigma}{4 \sigma^{2}}-\frac{\zeta_{5}^{2} \sigma \tau}{4 \sigma^{2} \tau^{2}}=\frac{\zeta_{2}^{2} \sigma+\zeta_{3}^{2} \tau-\zeta_{5}^{2}}{4 \sigma \tau}
$$

must be integral. 
By Theorem 5 we have $\zeta_{3}=x_{3} \sigma, \zeta_{5}=x_{5} \sigma$, and $\zeta_{2}^{2}+\left(x_{3}^{2} \tau-x_{5}^{2}\right) \equiv 0$ $(\bmod 4 \tau)$, which is equivalent to

$$
\zeta_{2}^{2}-x_{5}^{2} \sigma \equiv 0(\bmod \tau), \zeta_{2}^{2}+x_{3}^{2} \tau-x_{5}^{2} \equiv 0(\bmod 4) .
$$

Also

$$
\omega_{4}=\frac{\zeta_{2} i}{2 \tau}+\frac{x_{3} j}{2}+\frac{x_{5} i j}{2 \tau}
$$

so that, since $v_{2}=1+i / 2$ is in $T$, so is

$$
v_{2} \omega_{4}=\frac{\zeta_{2}}{4}+\frac{\zeta_{2}}{4 \tau} i+\frac{x_{3}+x_{5}}{4} j+\frac{x_{3} \tau+x_{5}}{4 \tau} i j .
$$

Since then $v_{2} \omega_{2}$ must have integral minimum equation, we see that $\zeta_{2} / 2$ is integral, $\zeta_{2}^{2} \equiv 0(\bmod 4), x_{3}^{2} \tau-x_{5}{ }^{2} \equiv 0(\bmod 2)$ and $x_{3} \equiv x_{5}(\bmod 2)$. But then $x_{3}^{2} \tau-x_{5}^{2} \equiv 0(\bmod 4)$ and $(44)_{2}$ is satisfied. We write $x_{3}=x_{5}+2 \lambda$.

Since $\sigma \equiv 4 \mu^{2}(\bmod \tau)$, we have $\zeta_{2}^{2} \equiv\left(2 \mu x_{5}\right)^{2}(\bmod \tau)$. But $-\tau$ is a prime so that $\zeta_{2} \equiv \pm 2 \mu x_{5}(\bmod \tau), \zeta_{2}= \pm 2 \mu x_{5}+y \tau$. Since $\zeta_{2}$ is even, so is $y=2 z$ and

$$
\omega_{4}=x_{5}\left(\frac{ \pm 2 \mu i+\tau j+i j}{2 \tau}\right)+\lambda j+z i
$$

which is evidently in $S$ or $\bar{S}$ according as the above sign is plus or minus. Hence $T$ is contained in $S$ or $\bar{S}$ as desired.

THEOREM 9. Every generalized quaternion division algebra in its canonical form of Theorem 4 has precisely two sets $S, \bar{S}$ of integral quantities satisfying $R, C, U^{\prime}, M$, and with

$$
\omega_{1}=1, \quad \omega_{2}=\frac{1+i}{2}, \quad \omega_{3}=j, \quad \omega_{4}=\frac{i(2 \mu+j)+\tau j}{2},
$$

as a tasis of $S$, while $\bar{S}$ has the corresponding basis

$$
\omega_{1}=1 \quad \omega_{2}=\frac{1+i}{2}, \quad \omega_{3}=j, \quad \bar{\omega}_{4}=\frac{i(-2 \mu+j)+\tau j}{2 \tau} .
$$

It is evident that the two sets $S, \bar{S}$ have almost identical properties and hence that there is essentially one set (either $S$ or $\bar{S}$ ) of integral quantities of $Q$.

The Institute for Advanced Study 